\title{
Simulation of phosphine flow in vertical grain storage: a preliminary numerical study
}

\author{
$\begin{array}{lll}\text { Z. Mat Isa } & \text { G. R. Fulford } & \text { N. A. Kelson } \\ & \end{array}$
}

(Received 28 January 2011; revised 5 September 2011)

\begin{abstract}
To fumigate grain stored in a silo, phosphine gas is distributed by a combination of diffusion and fan-forced advection. This initial study of the problem mainly focuses on the advection, numerically modelled as fluid flow in a porous medium. We find satisfactory agreement between the flow predictions of two Computational Fluid Dynamics packages, Comsol and Fluent. The flow predictions demonstrate that the highest velocity $(>0.1 \mathrm{~m} / \mathrm{s})$ occurs less than $0.2 \mathrm{~m}$ from the inlet and reduces drastically over one metre of silo height, with the flow elsewhere less than $0.002 \mathrm{~m} / \mathrm{s}$ or $1 \%$ of the velocity injection. The flow predictions are examined to identify silo regions where phosphine dosage levels are likely to be too low for effective grain fumigation.
\end{abstract}

http://anziamj . austms.org.au/ojs/index.php/ANZIAMJ/article/view/3933 gives this article, (c) Austral. Mathematical Soc. 2011. Published September 8, 2011. ISSN 1446-8735. (Print two pages per sheet of paper.) Copies of this article must not be made otherwise available on the internet; instead link directly to this URL for this article. 


\section{Contents}

1 Introduction

C760

2 Model equations and numerical solution $\quad$ C761

2.1 Model equations used for Comsol . . . . . . . . . . . . C762

2.2 Model equations used for Fluent . . . . . . . . . . . . . C763

2.3 Numerical solution methods . . . . . . . . . . . . . . C764

3 Results

C764

4 Conclusions

C770

References

C770

\section{Introduction}

The Australian grain industry seeks strategies that will kill $100 \%$ of insects in grain storage. In practice, a common method for killing insects is fumigation by phosphine gas as it is cheap, easy to use, and is a comparatively safe for most common stored grain commodities. There is currently worldwide acceptance of phosphine fumigation as a residue free treatment $[3,1]$. However, failed fumigation has been reported, and the ineffective spatial phosphine distribution in the grain storage is one of the factors contributing to the failure [5]. This, along with a rise in the number of phosphine resistant stored grain pests, means that a good understanding of how phosphine is distributed in grain storage is needed to ensure its long term use.

Phosphine gas has a specific gravity that is almost the same as air (air:1.0, $\mathrm{PH}_{3}: 1.17$ ) [5] and moves very slowly through the grain mass by diffusion if the air in the storage silo is not moving. Therefore, fan assisted fumigation is used to drive the phosphine gas through the grain from high to low pressure areas. The purpose of this work is to perform an initial study of this advection 
driven phosphine transport, and to this end we focus on a prototype geometry for a typical cylindrical farm silo. Knowledge of the flow patterns in the stored grain will, for example, give an indication of the zones in the grain storage that might provide areas of refuge for breeding insects.

Previous work related to this problem was conducted by Smith et al. [8, 9] who considered the advection-diffusion of $\mathrm{CO}_{2}$ in a cylindrical storage, assuming a uniform vertical flow. They obtained an analytic solution for the concentration using a perturbation approach based on small curvature of streamlines. A study of moisture transport coupled with heat transport in grain silos assuming Darcy flow in a porous medium was conducted by Singh and Thorpe [7] who numerically solved the governing equations using a finite difference approach. Their objective was to study the cooling of grain masses since stored product insects cannot thrive in low temperatures. Other research has been conducted by $\mathrm{Xu}$ et al. [10], who analytically studied the $\mathrm{CO}_{2}$ distribution in the bulk grain, again assuming Darcy flow.

We describe some initial results for the fan forced flow of phosphine into the base of a grain silo, numerically modelled as fluid flow in a porous medium. For comparison purposes, the flow is investigated using two CFD packages Comsol Multiphysics [4] and Ansys Fluent [6]. Comsol is a general purpose PDE solver based on the finite element method whereas Fluent is a CFD solver based on the finite volume method. The model equations used for each of the software packages are described and compared, and a detailed comparison of numerical results is presented. Implications of the results for phosphine fumigation of grain are discussed along with further work to be undertaken on this problem.

\section{Model equations and numerical solution}

The geometry chosen for this study is a vertical cylinder intended to model a storage facility having a radius $2 \mathrm{~m}$ and height $6 \mathrm{~m}$, typical of many domestic 
silos on farms. A $0.2 \mathrm{~m}$ radius inlet pipe is attached to the centre of the silo base. The resulting computational domain is axisymmetric, whereas practical silos may not be due to, for example, inlets located in the side walls. In view of this, a fully 3D solution methodology has been used in Fluent and Comsol as a precursor to future studies involving more complex domains. However, the current axisymmetric domain does permit a computationally less expensive 3D axisymmetric solution to be obtained for comparison purposes using Fluent (Comsol does not have this simplified modelling capability). Flow predictions are obtained for various phosphine gas and air mixtures pumped continuously at velocity $v_{0}=0.2 \mathrm{~m} / \mathrm{s}$ into the storage (filled with wheat). Mixtures of phosphine gas and carbon dioxide are also considered. Because the specific gravity of phosphine is similar to air, it is reasonable to neglect the effect of gravity on the flow. We also assume that the variation in the grain (wheat) temperature is negligible during fumigation [2] and that the pore size distribution is uniform with height. While some minor compaction is expected, the assumption of uniform pore size distribution is reasonable for an initial study.

\subsection{Model equations used for Comsol}

The mathematical model of the porous media flow solved using the Comsol Multiphysics package assumes gas flow through a grain bulk that satisfies Darcy's law $[9,10]$

$$
v=-\frac{k}{\mu} \nabla p
$$

with velocity $\boldsymbol{v}$, pressure $p$, grain permeability $k$ and dynamic viscosity $\mu$. Typically, for a pure phosphine gas in wheat grain, $k=0.578 \times 10^{-8} \mathrm{~m}^{2}[10]$ and $\mu=1.1 \times 10^{-5} \mathrm{~kg} \mathrm{~m}^{-1} \mathrm{~s}^{-1}$. By comparison, for pure $\mathrm{CO}_{2}$ gas, $\mathrm{k}=$ $2.5159 \times 10^{-8} \mathrm{~m}^{2}$ and $\mu=18.1 \times 10^{-6} \mathrm{~kg} \mathrm{~m}^{-1} \mathrm{~s}^{-1}$ [7]. In this initial study we also assume an incompressible flow, $\boldsymbol{\nabla} \cdot \boldsymbol{v}=0$, following Smith et al. [9, 8], which implies the pressure satisfies Laplace's equation

$$
\nabla^{2} p=0
$$


Equation (2) is solved subject to the following zero normal velocity boundary conditions on the vertical wall boundary

$$
\boldsymbol{\nabla} p \cdot \mathfrak{n}=0 \quad \text { on } \mathbf{r}=\mathrm{a},
$$

where $\boldsymbol{n}$ is the unit normal vector to the boundary and $\boldsymbol{a}$ is the radius of the silo. Phosphine gas flows through the grain surface at $z=h$ into the atmosphere. It is reasonable to assume that the gas pressure is constant at $z=h$, and if we assume Bernoulli's equation then $p=p_{a}-\rho v^{2} / 2$, where $p_{a}$ is the atmospheric pressure and $\rho$ is the density. However, $v$ will be small compared to the inlet velocity, so it is reasonable to linearise around the velocity and therefore approximate the boundary condition with

$$
\mathrm{p}=\mathrm{p}_{\mathrm{a}} \quad \text { on } z=\mathrm{h} .
$$

On the base of the cylinder,

$$
\nabla p \cdot \mathfrak{n}=\left\{\begin{array}{ll}
-\mu v_{0} / k, & r<b, \\
0, & r>b,
\end{array} \quad \text { at } z=0,\right.
$$

where $\mathrm{b}=0.2 \mathrm{~m}$ is the inlet radius and $\nu_{0}=0.2 \mathrm{~m} / \mathrm{s}$ is the velocity injection of the phosphine at the inlet.

\subsection{Model equations used for Fluent}

In contrast to Comsol, Fluent solves the usual Navier-Stokes equations which include conservation equations for mass and momentum, along with an additional resistance term $\mathbf{S}$ to account for the porous medium,

$$
\rho(\boldsymbol{v} \cdot \nabla) \boldsymbol{v}=\mu \nabla^{2} \boldsymbol{v}+\mathbf{S}, \quad \mathbf{S}=-\frac{\mu}{\mathrm{k}} \nabla p .
$$

In Fluent, the no-slip wall condition, $\boldsymbol{v}=\mathbf{0}$, replaces boundary conditions (3) and (4) for $\boldsymbol{r}>\boldsymbol{b}$. For this flow, the inertia terms are typically small over most of the domain. Neglecting these in (5), taking the divergence of the remaining terms, and using the continuity equation results in an equation which is mathematically equivalent to (2). 


\subsection{Numerical solution methods}

In this work all simulations were run on a Windows XP PC with $3 \mathrm{Ghz}$ Intel(R) Core(TM)2 Duo (E8400). For the 3D computational modelling, an unstructured tetrahedral mesh, with local refinement near the inlet, is used in both Comsol and Fluent. Overall, the surface meshing used for both is similar. Inside the flow volume, the Fluent simulation uses 180, 737 cells whereas the Comsol simulation uses 62,898 cells, the latter being about one third of the former due to software memory limitations. Throughout the computational domain, Comsol uses an iterative GMREs linear system solver and geometric multi-grid pre-conditioner to solve for the pressure field, along with post processing to calculate the velocity. Fluent instead uses an iterative Navier-Stokes solver which separately solves for the pressure and velocity at each step. For the spatial discretisation, first order upwinding was available for use in both packages, with second order upwinding available in Fluent only. The use of higher order upwinding did not affect the results significantly, as expected, since velocities are small.

In the Comsol simulation, the discontinuous velocity at the bottom surface, $z=0$, due to gas injection, $v_{0}$, is replaced by the hyperbolic tangent function

$$
v_{0} \times 0.5\{1-\tanh [\alpha(r-b]\},
$$

with $\alpha=50$. The use of this expression aims to reduce any local numerical instability caused by the flow discontinuity across the inlet at $r=b$.

\section{$3 \quad$ Results}

The overall predicted flow features are illustrated in Figure 1 which shows the streamlines and a contour plot of the velocity magnitude through a silo $2 \mathrm{D}$ vertical slice. The results shown are obtained using the full $3 \mathrm{D}$ solver capability of Fluent, with corresponding results obtained using Comsol being 
indistinguishable by eye (not shown for brevity). Overall, the flow moves upward from the inlet at the base $(z=0)$ towards the grain surface, and axial symmetry appears to be preserved throughout the domain. The gas velocity decreases rapidly: over a radial distance of $1 \mathrm{~m}$ from the inlet it decreases to less than $1 \%$ of the velocity injection $v_{0}$. From about a height of $1 \mathrm{~m}$ and above, the flow is spread the full extent of the silo radius and the streamlines are almost parallel. Sample computations with viscosity, $\mu$, and permeability, $k$, changed from pure phosphine to pure carbon dioxide result in very similar velocity and streamline patterns, suggesting that the flow is not overly sensitive to changes in these parameter values. Although not easily seen from the figure, the regions of lowest velocity are near the corners where the base plate and vertical wall meet. Specifically, the velocity magnitude is less than $0.004 \%$ of $v_{0}$ in the flow region located at the base of the silo and within $0.1 \mathrm{~m}$ of the vertical silo wall. This very slow flow region is likely to be where the distribution of phosphine gas through diffusion plays an important role. It may also be an area of refuge for insects against any grain fumigation treatment of insufficient duration to achieve critical dosage levels throughout the entire storage silo.

Additional flow detail for the 3D simulations is provided in Figure 2 and Figure 3, where velocity profiles predicted by both Comsol and Fluent are plotted at different locations. Along the vertical symmetry line of the silo, the axial velocity $v_{z}$ decreases rapidly with height from its inlet value at $z=0$ before tending to approximately constant, as portrayed in Figure 2(a). Physically, this demonstrates the inability of the gas to penetrate quickly to the height of the grain mass. Along this same vertical symmetry line, the transverse velocity $v_{x}$ is expected to be zero for axisymmetric flow. However, in Figure 2(b), small nonzero transverse velocities are observed close to the inlet in both the Comsol and Fluent predictions, with these being more pronounced for Comsol.

In Figure 3, the axial velocity profiles across the inlet plane and downstream at height $z=3$ are given. As shown in Figure 3(a), essentially top hat inlet profiles are imposed for the axial velocity in both Comsol and Fluent, with 


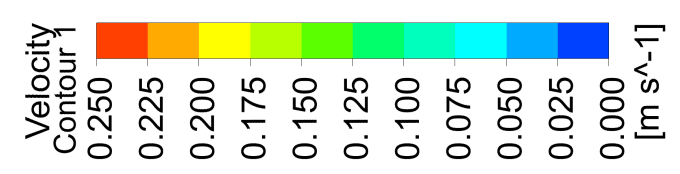
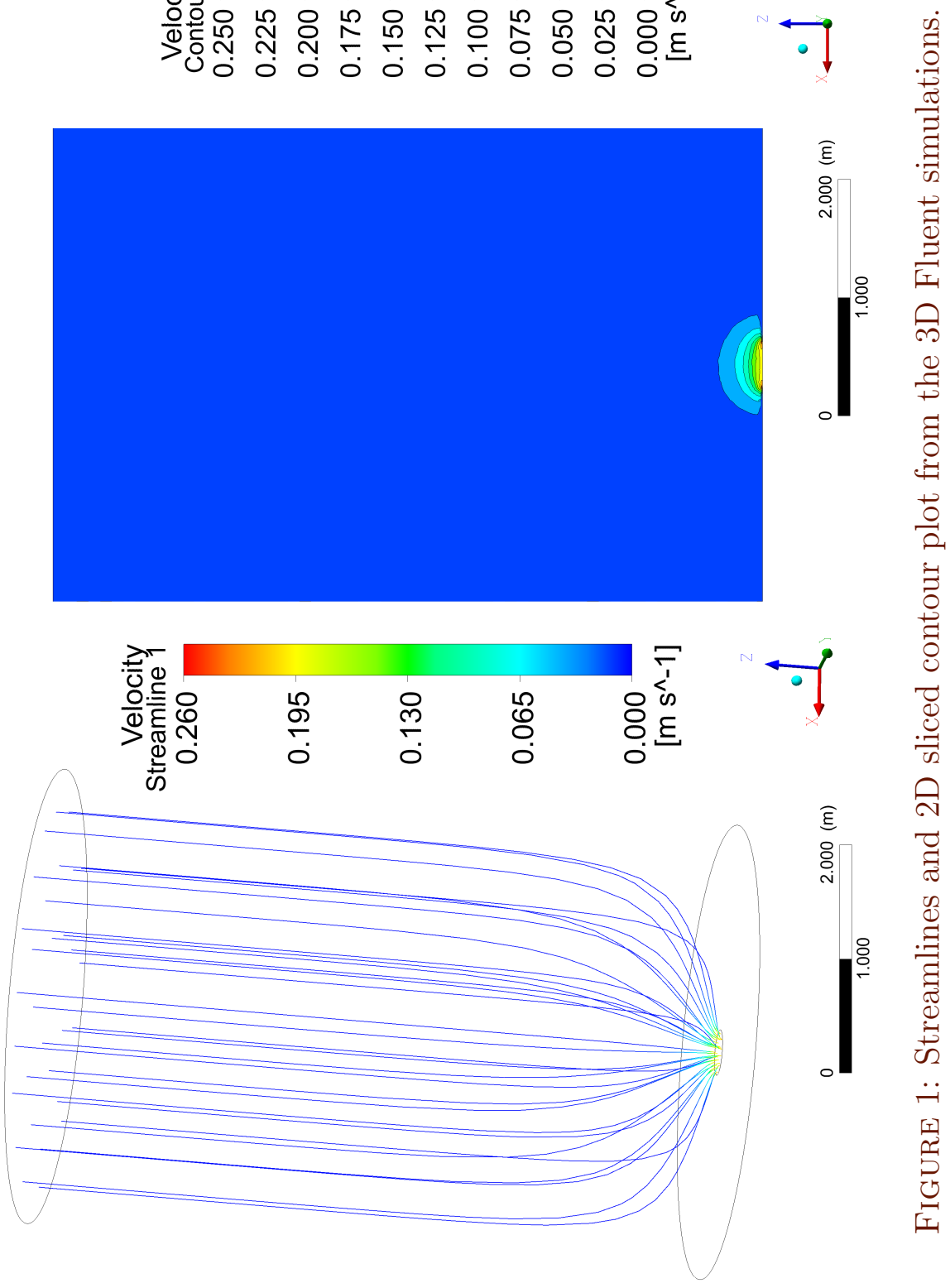


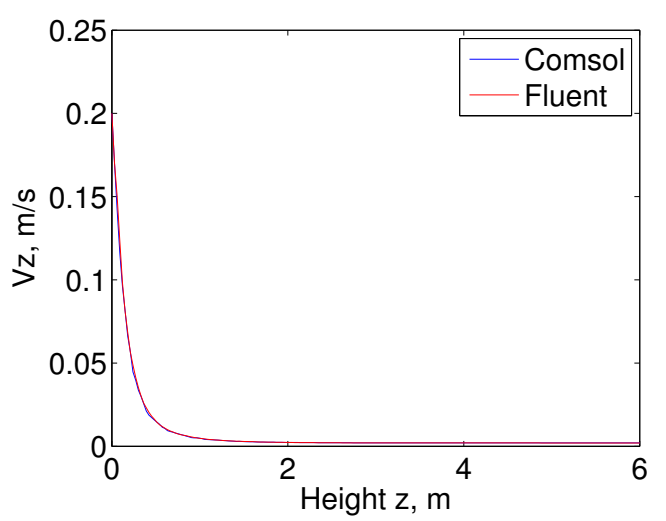

(a)

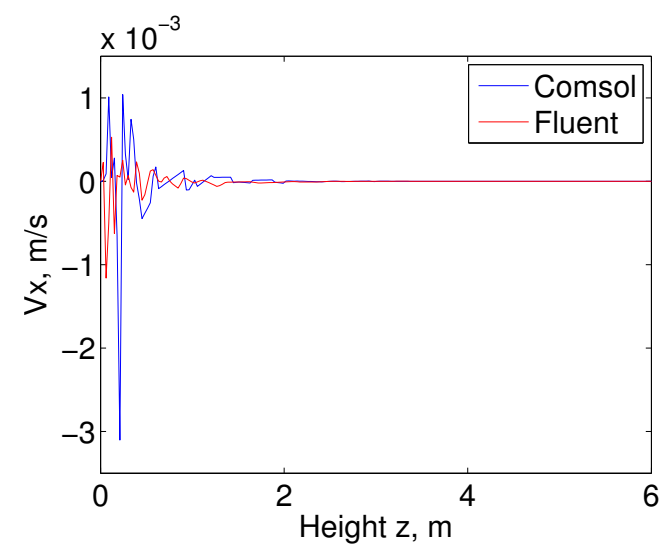

(b)

FIGURE 2: Variation with height of (a) axial velocity $v_{z}$, and (b) transverse velocity $v_{x}$ along the symmetry line of the silo.

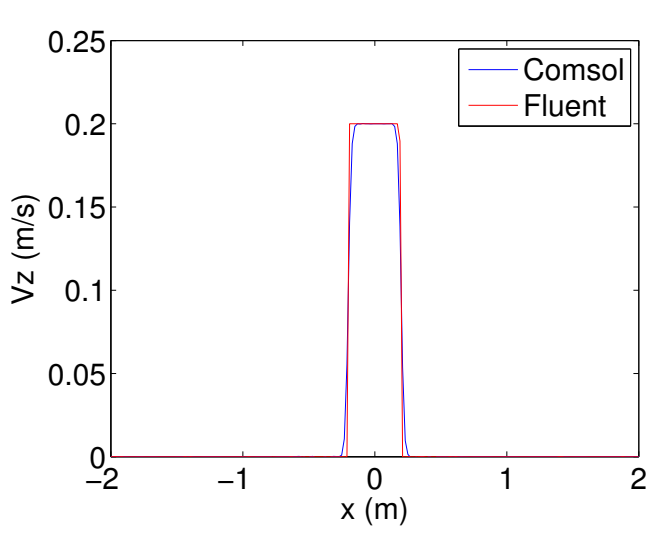

(a)

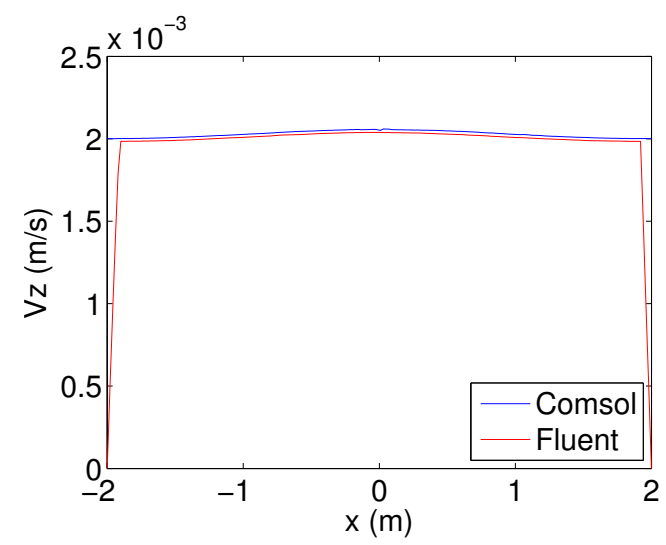

(b)

FiguRE 3: Axial velocity profile $v_{z}$ (a) at height $z=0$, and (b) $z=3$. 
some smoothing of the Comsol inlet profile due to the hyperbolic tangent function across the inlet. Despite this smoothing, nonzero variations in the transverse velocity component across the inlet are found in the Comsol predictions and are attributable to the simplified modelling. In Figure 3(b) the downstream behaviour of the flow at $z=3$ is different for the two models when near the wall, with the Fluent simulations imposing the no-slip condition on the vertical walls, whereas in Comsol only the normal component of the velocity is required to be zero. Nevertheless, away from the narrow near wall region, an essentially uniform unidirectional axial velocity is predicted by both solvers across the entire silo width. Although not shown for brevity, the corresponding predicted transverse velocity components are close to zero. Hence, in the upper regions of the stored grain, it is mainly the magnitude of the axial velocity that determines the rate of phosphine distribution.

As previously mentioned, a simplified 3D axisymmetric formulation of the governing equations is available in Fluent. This permits flow predictions on a rectangular grid corresponding to a vertical slice of the 3D cylindrical storage, at greatly reduced computational cost. Here, we investigate the convergence behaviour of the axisymmetric Fluent model as the grid is refined using four different grid sizes. Sample results are given in Table 1 for the relative error of the three coarsest axisymmetric grids and the Fluent full $3 \mathrm{D}$ simulation results, relative to the finest grid size used $(300 \times 900)$ in the axisymmetric modelling. As expected, the relative error for the peak velocities decreases with increasing number of elements, $\mathrm{N}$, as do the relative errors for the velocities at the point $(0.2,0,1)$. Assuming a form $E=\mathrm{kN}^{\alpha}$ then regression on the logarithm of the variables gives an exponent $\alpha \simeq-1.2$ for the peak axial velocity and $\alpha=-1.7$ for both velocity components at the point $(0.2,0,1)$. This indicates better than quadratic convergence based on a typical cell length scale $1 / \sqrt{\mathrm{N}}$. However, for the peak radial velocity, the convergence is slower with $\alpha \simeq-0.7$. While there is reasonable accuracy for the axial peak velocities for the 3D simulation, the tabulated data suggest that further grid refinement is required in the full 3D modelling if more accurate predictions for the transverse velocity components are desirable. 
TABLE 1: Relative errors of maximum velocities from axisymmetric Fluent model predictions on three different grids, relative to axisymmetric $300 \times 900$ results. Also shown are relative errors for velocities at sample point $(0.2,0,1)$ and the number of cells $\mathrm{N}$. Corresponding results for the full 3D Fluent simulation are also given.

\begin{tabular}{cccccc}
\hline grid size & $\mathrm{N}$ & \multicolumn{4}{c}{ \% error } \\
& & $\max v_{z}$ & $\max v_{x}$ & $v_{z}(0.2,0,1)$ & $v_{x}(0.2,0,1)$ \\
\hline $3 \mathrm{D}$ & $1.8 \times 10^{5}$ & 1.9 & 29.6 & 2.9 & 1.5 \\
$50 \times 150$ & $7.5 \times 10^{3}$ & 8.6 & 44 & 0.5 & 1.1 \\
$100 \times 300$ & $3 \times 10^{4}$ & 3.4 & 26 & 0.1 & 0.25 \\
$200 \times 600$ & $1.2 \times 10^{5}$ & 0.8 & 9.2 & 0.02 & 0.04 \\
\hline
\end{tabular}

As part of this initial study, we also sought an analytic solution for the axisymmetric version of the model equations used by Comsol and were able to find a closed form series solution involving an infinite Fourier-Bessel series,

$$
p(r, z)=D z+E+\sum_{m=1}^{\infty} J_{0}\left(\frac{\alpha_{m}}{a} r\right)\left[A_{m} \cosh \left(\frac{\alpha_{m}}{a} z\right)+B_{m} \sinh \left(\frac{\alpha_{m}}{a} z\right)\right]
$$

with

$$
\mathrm{D}=-\frac{\mu v_{0} b^{2}}{k a^{2}}, \quad E=p_{a}-D h
$$

and

$$
B_{m}=-\frac{2 \mu b v_{0}}{k} \frac{J_{1}\left(\alpha_{m} b / a\right)}{\alpha_{m}^{2}\left[J_{0}\left(\alpha_{m}\right)\right]^{2}}, \quad A_{m}=\frac{-B_{m} \sinh \left(\alpha_{m} h / a\right)}{\cosh \left(\alpha_{m} h / a\right)} .
$$

A detailed analysis of this closed form solution, including, for example, convergence behaviour, has not yet been undertaken and remains the subject of future work. 


\section{Conclusions}

We used CFD modelling with Comsol and Fluent to perform a simulation for phosphine gas flow in a cylindrical grain storage silo. On the whole, Comsol and Fluent predict similar flow behaviour with satisfactory agreement. The flow predictions demonstrate that the region with highest velocity $(>0.1 \mathrm{~m} / \mathrm{s})$ occurs within a $0.2 \mathrm{~m}$ radius distance from the inlet and reduces drastically over one metre of silo height. A very slow flow region was identified at the base of the silo and within $0.1 \mathrm{~m}$ of the silo wall, where diffusion may play an important role in the phosphine gas distribution. Additionally, this region may also be a refuge for insects against any grain fumigation treatments.

In future work, we will consider the advection-diffusion transport of the phosphine, using the flow results in this article as an input. More complex grain storage geometries will also be explored. Of significant interest to the grain industry is whether 'pockets' exist where insects can survive more easily. The critical parameters for such regions include both the local concentration as well as the time of exposure, so some modelling of the mortality of insects should be coupled to this model to provide useful insights for future design of cost effective fumigation strategies.

\section{References}

[1] K. Bullen, Insect control in stored grain, Technical report, DPI\&F, Plant Science, Toowoomba, Queensland, 2007. http://www.dpi.qld.gov.au/26_6263.htm C760

[2] J. Canchun, D. W. Sun, and C. Cao, Computer simulation of temperature changes in a wheat storage bin, Journal of Stored Products Research, 37, 165-177, 2001. doi:10.1016/S0022-474X(00)00017-5 C762 
[3] P. J. Collins, G. J. Daglish, M. K. Nayak, P. R. Ebert, D. Schlipalius, W. Chen, H. Pavic, T. M. Lambkin, R. Kopittke, and B. W. Bridgeman, Combating resistance to phosphine in Australia, Proc. Int. Conf. Controlled Atmosphere and Fumigation in Stored Products, Executive Printing Services, 593-607, 2001. http://www.ftic.info/CAFSITE/CAFPDF/60.pdf C760

[4] Comsol (2008). Comsol multiphysics user's guide. http://www.comsol.com C761

[5] P. W. Flinn and C. Reed, Effects of outside air temperature on movement of phosphine gas in concrete elevator bins, Proceedings of the International Conference on Controlled Atmosphere and Fumigation in Stored Products, 704-706, 2008. C760

[6] Fluent, Ansys inc. http://www.ansys.com C761

[7] A. K. Singh and G. R. Thorpe, A solution procedure for three-dimensional free convective flow in peaked bulks of grain, Journal of Stored Products Research, 29, 221-235, 1993. doi:10.1016/0022-474X(93)90004-N C761, C762

[8] E. A. Smith, D. S. Jayas, and A. D. Ville, Modelling the flow of carbon dioxide through beds of cereal grains, Transport in Porous Media, 44, 123-144, 2001. doi:10.1023/A:1010760508392 C761, C762

[9] E. A. Smith and D. S. Jayas, Modeling the movement of fumigant gas within grain beds, American Society of Agricultural Engineers, 44(3), 661-667, 2001. http://asae.frymulti.com C761, C762

[10] S. Xu, D. S. Jayas, N. D. G. White, and W. E. Muir, Momentum-diffusive model for gas transfer in granular media, Journal of Stored Products Research, 38, 455-462, 2002.

doi:10.1016/S0022-474X(01)00048-0 C761, C762 


\section{Author addresses}

1. Z. Mat Isa, Discipline of Mathematical Sciences, Queensland University of Technology, Brisbane 4103, Australia. mailto:zaiton.matisa@student.qut.edu.au

2. G. R. Fulford, Discipline of Mathematical Sciences, Queensland University of Technology, Brisbane 4103, Australia. mailto:g.fulford@qut.edu.au

3. N. A. Kelson, High Performance Computing and Research Support Group, Division of Technology, Information and Learning Support, QUT, Brisbane 4103, Australia. mailto:n.kelson@qut.edu.au 